\title{
Nanostructured poly(benzimidazole) membranes by N-alkylation
}

\author{
P. Haro Dominguez, K. Grygiel, J. Weber* \\ Max Planck Institute of Colloids and Interfaces, Department of Colloid Chemistry, Research Campus Golm, D-14424 \\ Potsdam, Germany
}

Received 16 May 2013; accepted in revised form 31 August 2013

\begin{abstract}
Modification of poly(benzimidazole) (PBI) by N-alkylation leads to polymers capable of undergoing microphase separation. Polymers with different amounts of $\mathrm{C}_{18}$ alkyl chains have been prepared. The polymers were analyzed by spectroscopy, thermal analysis, electron microscopy and X-ray scattering. The impact of the amount of alkyl chains on the observed microphase separation was analyzed. Membranes prepared from the polymers do show microphase separation, as evidenced by scattering experiments. While no clear morphology could be derived for the domains in the native state, evidence for the formation of lamellar morphologies upon doping with phosphoric acid is provided. Finally, the proton conductivity of alkyl-modified PBI is compared with that of pure PBI, showing that the introduction of alkyl side chains does not result in significant conductivity changes.
\end{abstract}

Keywords: polymer membranes, molecular engineering, nanomaterials

\section{Introduction}

Proton exchange membrane fuel cells (PEMFCs) received a great deal of interest in recent years as they provide an alternative to classic combustion engines. Nafion ${ }^{\circledR}$ can still be considered as a benchmark for proton exchange membranes, despite the fact that its development by DuPont was more than 50 years ago. Ever since then, big efforts have been done by different research groups to understand the characteristic features of Nafion [1-4], which makes it superior to other systems. The microphase-separated microstructure of Nafion, providing mechanical strength together with well-connected water domains that facilitate proton-transport, has been identified as the reason for the superior performance of Nafion. The rather high price of Nafion has led researchers to look for alternative ionomer membranes. This search took almost always the possibil- ity of the polymers to undergo microphase separation into account.

Next to ionomers, acid-base complexes have been regarded as alternatives to Nafion. The probably most prominent example of this class of materials is phosphoric acid (PA) doped polybenzimidazole (PBI) $[2,5,6]$. In this material, PBI provides the mechanical support of the membrane and acts as a basic anchor to the phosphoric acid, which is mainly responsible for the high proton conductivity. One advantage of the PBI system is its easy operability up to high temperatures $\left(150-180^{\circ} \mathrm{C}\right)$, which is not easily possible with Nafion membranes. Operation at such high temperatures results in higher tolerance against fuel impurities and an easier heat management and is hence regarded as beneficial. However, the PA-PBI system lacks to the best of our knowledge any self-organization into a microphase-sepa-

\footnotetext{
${ }^{*}$ Corresponding author, e-mail: jens.weber@mpikg.mpg.de (C) BME-PT
} 
rated structure known from the ionomers. Typically, the PBI chains interrupt the proton conduction pathways of the PA, which consist mainly of dynamic hydrogen-bonded structures [7]. This can be partly overcome by creating nanostructured PA-PBI systems, which do indeed show higher proton conductivities compared to homogenous systems [8]. In earlier work, mesoporous PBI was prepared by templating [9], and the pores were backfilled by PA, which is overall a rather time-consuming synthesis pathway, which is hard to implement in membrane technology. There are a few other attempts to achieve nanostructural control, which were reported recently, including (multi)block copolymer synthesis and generally, a positive effect on the conductivity was found but no detailed structural information (e.g. domain size) was provided so far [10-12].

Inspired by recent attempts to use graft copolymers to induce microphase-separated structures for organic-electronics applications [13], we aim to synthesize poly[2,2'-(m-phenylene)-5,5'bibenzimidazole] (PBI) based graft-copolymers that can undergo microphase separation. Within this communication, we present the grafting of PBI with long $\left(\mathrm{C}_{18}\right)$ alkyl chains following modification protocols presented by Gieselman and Reynolds [14]. The modification of PBIs by N-alkylation was reported by previously by Klaehn et al. [15], who did however not study the mesostructure of the resulting polymers. The modification of so-called ABPBI, i.e. poly(2,5-benzimidazole) by $\mathrm{N}$-alkylation for gas permeation membranes was reported by Kumbharkar and Kharul [16], but again no analysis of any microphase separation was studied. Finally, a very recent study by Jana and coworkers came to our attention, which dealt with the N-alkylation of PBI. Alkyl chains up to $\mathrm{C}_{16}$ length were introduced [17]. The study analysed the impact of the alkyl chain length and reported improved solubility while maintaining satisfactory proton conductivity.

There is however no detailed study on the microphase separation of alkylated PBI available yet. It is hence within the focus of the current manuscript to provide an analysis of the microstructure of the resulting polymers in dry and phosphoric acid doped state. Characterization will be done by means of small-angle and wide-angle X-ray scattering (SAXS and WAXS) and electron microscopy. First conductivity data will also be reported.

\section{Experimental}

\subsection{Materials and methods}

3,3'-diaminobenzidine (DAB, 99\%, D12384) and diphenyl isophthalate (DPI, 99\%, 411698) were purchased from Aldrich via Sigma-Aldrich (Steinheim, Germany) and used without further purification. Sodium hydride (NaH, 95\%, 223441) and dry NMP $(99.5 \%, 328634)$ and dry N,N-dimethylacetamide (DMAc, 99.8\%, 271012) were also purchased from Sigma-Aldrich. 1-Bromooctadecane ( $>97 \%, 199494)$ was purchased from Aldrich. Other solvents used for workup or syntheses under ambient conditions, such as methanol $(\mathrm{MeOH}$, Merck 1.06008.9180, Darmstadt, Germany), diethylether (VWR, 23811.361; Leuven, Belgium), DMAc (Alfa Aesar; A10924, Karlsruhe, Germany) were of analytical grade.

${ }^{1} \mathrm{H}-\mathrm{NMR}$ measurements were carried out using a Bruker DPX-400 spectrometer (Bruker, Germany) operating at $400.1 \mathrm{MHz}$. As solvents, deuterated chloroform $\left(\mathrm{CDCl}_{3}, 151823\right)$, deuterated dimethylsulfoxide (DMSO- $d 6,522120$ ), deuterated tetrahydrofuran (THF-d8, 184314) (all Sigma-Aldrich) or mixtures thereof were used. Calibration was carried out using signals corresponding to non-deuterated solvent ( $\mathrm{CDCl}_{3}: 7.26 \mathrm{ppm}$; DMSO-d6: $\left.2.50 \mathrm{ppm}\right)$. Fourier-transform infrared (FT-IR) spectra were collected with a Varian 1000 Scimitar FT-IR spectrometer (FTS-1000, Varian, Berlin, Germany), equipped with an attenuated total reflection (ATR) setup.

Gel permeation chromatography (GPC, Thermo Separation Products (TKA), Niederelbert, Germany) in $0.5 \mathrm{wt} \% \mathrm{LiBr}$ in $\mathrm{N}$-methylpyrrolidon (NMP) as the eluent was performed with a system containing PSS (Mainz, Germany) GRAM 100 and $1000 \AA$ columns. It provides simultaneous ultraviolet (UV) and refractive index (RI) detection. $100 \mu \mathrm{L}$ were injected and separated at $70^{\circ} \mathrm{C}$ and a flow of $0.8 \mathrm{~mL} \cdot \mathrm{min}^{-1}$. GPC in $0.5 \mathrm{wt} \% \mathrm{LiBr}$ in DMSO as the eluent was performed with a GPC system (Thermo Separation Products (TKA), Niederelbert, Germany) containing two PSS GRAL LIN columns. It provides simultaneous UV and RI detection. $100 \mu \mathrm{L}$ were injected and separated at $70^{\circ} \mathrm{C}$ and a flow of $1.0 \mathrm{~mL} \cdot \mathrm{min}^{-1}$. Samples were filtered through $0.45 \mu \mathrm{m}$ syringe filters prior to use.

WAXS measurements were performed with a D8 Advance machine from Bruker Instruments (Bruker AXS, Karlsruhe, Germany). The radiation was of the wavelength of $\mathrm{CuK}_{\alpha}(0.1542 \mathrm{~nm})$. Measurements 
were done in reflection geometry. Samples were measured as fine powders (or films respectively) on a silicon sample holder.

SAXS on solid samples was measured with a Rotating Anode Instrument $\left(4 \mathrm{~kW}, \mathrm{CuK}_{\alpha}\right.$, Enraf-Nonius, Germany) with point collimation and MARCCD detector (pixel size 79, Marresearch, Norderstedt, Germany) at room temperature and a distance of $74 \mathrm{~cm}$ between detector and sample. 2D diffraction pictures were corrected for background scattering and transformed into 1D radial averages of the scattering intensity using the Fit2D program. Calibration was done using silver behenate.

Thermogravimetric Analysis (TGA) was carried out on a Netzsch TG 209 F1 (Netzsch, Selb, Germany) at $10 \mathrm{~K} \cdot \mathrm{min}^{-1}$ under either synthetic air or nitrogen atmosphere.

Scanning electron microscopy (SEM) pictures were taken with a Gemini Leo 1550 (Zeiss AG, Jena, Germany) microscope at $3 \mathrm{kV}$. The samples were loaded on carbon coated stubs and coated by sputtering with $\mathrm{Au} / \mathrm{Pd}$ alloy prior to imaging.

Proton Conductivity: Resistance measurements were performed using a Solartron SI1260 Impedance/Gain Phase analyzer and Solartron SI1287 Electrochemical interface (AC amplitude: $10 \mathrm{mV}$; DC potential versus open circuit: $100 \mathrm{mV}$; frequency range: $0.5 \mathrm{MHz}$ to $0.1 \mathrm{~Hz}$; Solartron Analytical, AMETEK GmbH, Meerbusch, Germany). Membrane disks of $6 \mathrm{~mm}$ diameter were contacted with Pt-foils of the same diameter and measurements were performed under closed atmosphere at room temperature $\left(22.5^{\circ} \mathrm{C}, 16 \%\right.$ outer humidity). The membranes were dried under vacuum at $60^{\circ} \mathrm{C}$ for 20 hours prior to measurements. The proton conductivity $\sigma$ was calculated from the resistance $\kappa$ by: $\sigma=h \cdot(\kappa A)^{-1}$, where $A$ is the surface area of the films $\left(0.2827 \mathrm{~cm}^{2}\right)$ and $h$ is the film thickness (determined using a vernier scale).

\subsection{Synthesis of $\mathbf{N}$-alkylated PBI}

The parent PBI was synthesized using standard protocols developed by Vogel and Marvel [18], and purified from insoluble byproducts by hot filtration from DMAc followed by precipitation and washing by methanol. In a typical alkylation experiment, $0.5 \mathrm{~g}$ of PBI were dissolved in anhydrous NMP at $80^{\circ} \mathrm{C}$ and cooled to $R T$. The required amount of sodium hydride was added under inert atmosphere and the mixture was heated to $80^{\circ} \mathrm{C}$ for $3 \mathrm{~h}$ under inert atmosphere. The required amount of 1-bromooctadecane was slowly added afterwards and the reaction was allowed to proceed at $80^{\circ} \mathrm{C}$ for $24 \mathrm{~h}$. The polymer was precipitated by $\mathrm{MeOH}$ after cooling to $R T$ and washed once with diethylether and three times with $\mathrm{MeOH}$ before dried under vacuum at $100^{\circ} \mathrm{C}$.

\section{Results and discussion}

\subsection{Chemical and structural characterisation}

PBI was synthesized by classic melt polycondensation, as introduced by Vogel and Marvel [18]. After solid-state curing at $400^{\circ} \mathrm{C}$, the crude product was stirred in hot DMAc (containing $0.5 \mathrm{wt} \% \mathrm{LiCl}$ ) for $20 \mathrm{~h}$, followed by hot filtration in order to exclude non-soluble byproducts. The chemical identity of the parent PBI was verified by FTIR and ${ }^{1} \mathrm{H}-\mathrm{NMR}$ spectroscopy. FTIR spectroscopy could clearly show the characteristic peaks of PBI at 1620, 1443 and $1280 \mathrm{~cm}^{-1}, 800$ and $700 \mathrm{~cm}^{-1}$ in accordance with literature [19]. The ${ }^{1} \mathrm{H}-\mathrm{NMR}$ spectra prove the expected structure of the polymer. At $13.3 \mathrm{ppm}$ the prominent $\mathrm{NH}$-protons on the benzimidazole appear, while the peaks between 9.2 and $7.6 \mathrm{ppm}$ can be assigned to the aromatic protons in the main backbone according to reported literature $[17,20]$. Reaction of deprotonated PBI with octadecyl bromide $\left(\mathrm{C}_{18}-\mathrm{Br}\right)$ was performed at $75^{\circ} \mathrm{C}$ for 3 days. Deprotonation of PBI was done by reaction with $\mathrm{NaH}$ in dry NMP at $75^{\circ} \mathrm{C}$ for 3 hours. A slight excess of $\mathrm{NaH}$ with respect to the targeted conversion was always used. We aimed at modification of PBI with $0.5,1$ and 2 equivalents of $\mathrm{C}_{18}$ - $\mathrm{Br}$ per repeat unit (RPU) in order to study the influence of the amount of alkyl chains on the microphase separation. Samples were named PBI-C $\mathrm{C}_{18^{-}} 1$ to $\mathrm{PBI}-\mathrm{C}_{18^{-}} 3$ where PBI$\mathrm{C}_{18}-1$ is the PBI with lowest degree of functionalization. The degree of alkylation (D.A.) was calculated based on NMR analysis of the resulting polymers (see Table 1 and Figure 1).

The calculation is based on the ratio between aromatic backbone $\mathrm{H}$ atoms, which are set to 10 protons, and the methylene protons next to the imidazolium $\mathrm{N}$ atom (found at $4.5 \mathrm{ppm}$ ). The methylene protons of the $\mathrm{N}-\mathrm{CH}_{2}-\mathbf{C H}_{2}-$ methylene group (at $1.83 \mathrm{ppm}$ ) can also be used for comparison and gave similar results. The NMR spectrum of the modified polymers showed a more complex aromatic region, which can be explained by the more complex chemical environment, which is intro- 
Table 1. Molecular characteristics of the parent PBI and the alkyl-modified PBI

\begin{tabular}{|c|c|c|c|c|c|c|}
\hline Entry & D.A. target & $\begin{array}{c}\text { D.A. } \\
\text { ('H-NMR) }\end{array}$ & $\begin{array}{l}\text { wt\% alkyl } \\
\text { (NMR) }\end{array}$ & $\begin{array}{c}\text { wt\% alkyl } \\
\text { (TGA) }\end{array}$ & $\begin{array}{c}\mathbf{M w}^{\mathbf{a})} \\
{\left[\mathbf{g} \cdot \mathbf{m o l}^{-1}\right]}\end{array}$ & $\oplus^{\text {a) }}$ \\
\hline PBI & - & - & - & & 45000 & 2.6 \\
\hline PBI-C $18-1$ & 0.5 & 0.44 & 25.6 & 18 & 52550 & 1.9 \\
\hline PBI-C $18-2$ & 1 & 0.68 & 30.4 & 28 & 53720 & 2.1 \\
\hline $\mathrm{PBI} \mathrm{C}_{18}-3$ & 2 & 1.18 & 36.4 & 42 & 62000 & 2.6 \\
\hline
\end{tabular}

a) determined by GPC at $70^{\circ} \mathrm{C}$, eluent: NMP, polystyrene (PS) standards
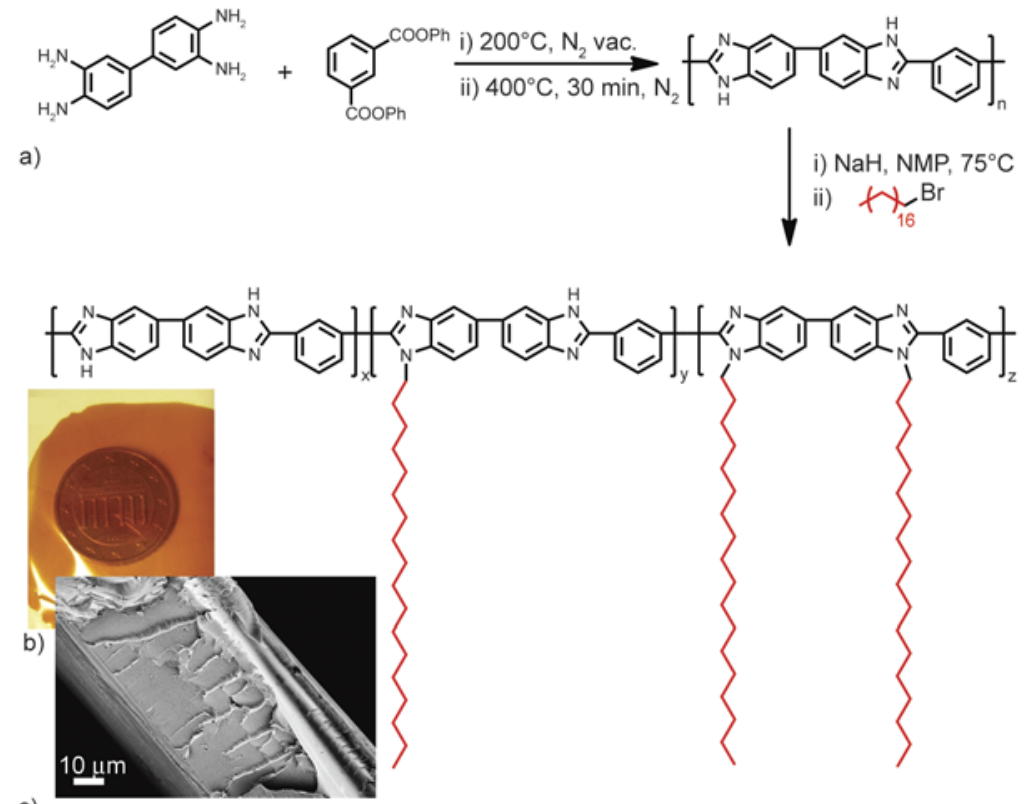

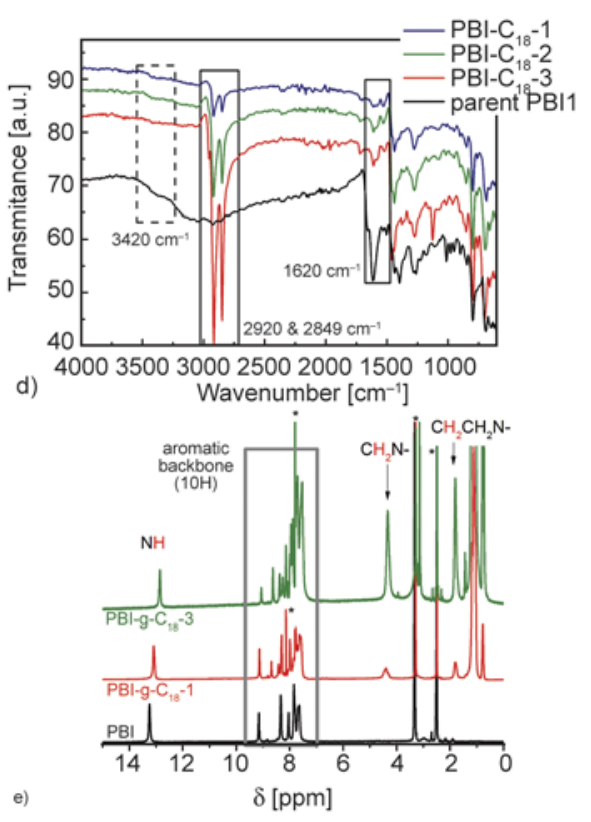

Figure 1. a) chemical structure and synthesis scheme of PBI and PBI-C 18 ; b) photograph of a PBI- $\mathrm{C}_{18}-3$ membrane and c) corresponding SEM micrograph (cross-section); d) FT-IR spectra of PBI and modified PBI, please note that the overly prominent band around $1650-1620 \mathrm{~cm}^{-1}$ in the case of pure PBI and (to lower extent also for PBI-g$\mathrm{C}_{18}-1 /-2$ ) is due to an overlap with solvent residues (DMAc); e) representative ${ }^{1} \mathrm{H}-\mathrm{NMR}$ spectra of PBI and PBI$\mathrm{C}_{18}-1$ and $\mathrm{PBI}-\mathrm{C}_{18^{-}} 3$, please note that the spectra were taken in different solvents/solvent mixtures, which explains the shift of the $\mathrm{NH}$ proton, * marks solvent peaks

duced upon grafting of alkyl chains to the imidazole units. Overall, the spectra agree well with spectra of short-chain alkyl modified PBI reported by Maity et al. [17]. The D.A. calculated from NMR results were furthermore supported by thermal gravimetric analysis (TGA), see below.

The reactions did not result in full conversions, i.e. the experimentally achieved D.A. was always lower than the targeted value. This is not unexpected, as full conversion in grafting-onto is typically hard to achieve, due to e.g. steric reasons. This becomes obvious especially for the case of targeted full alkylation, where the PBI backbone chain would have to stretch to allow all alkyl chains to be attached, resulting in a brush-like morphology. Hence, the degree of success of the grafting reaction dropped from $88 \%$ for $\mathrm{PBI}-\mathrm{C}_{18}-1$ to $67.5 \%$ for $\mathrm{PBI}-\mathrm{C}_{18}-2$ and $59 \%$ for $\mathrm{PBI}-\mathrm{C}_{18}-3$. These values can be translated into the weight fraction of the alkyl component within the graft copolymers, which is found to be 25.6, 30.4 and $36.4 \mathrm{wt} \%$ for PBI-C ${ }_{18}-1$ to PBI- $\mathrm{C}_{18}-3$, respectively. It should be noted that full conversion could be reached in comparable $\mathrm{N}$-alkylation reactions, given that a significant excess of the alkyl compound was used [15]. The degree of alkylation reached within this study agrees reasonable with results obtained by Maity et al. [17].

Overall, alkylation resulted in a better solubility of the polymers in common organic solvents. For instance, PBI-C $1_{18}-3$ is soluble in THF and $\mathrm{CHCl}_{3}$, which are typically not good solvents for PBI. Transparent films could be casted from $10 \mathrm{wt} \%$ solutions of PBI-C ${ }_{18}-3$ in THF or $\mathrm{CHCl}_{3}$ after filtration. The increased solubility was only observed for the highest degree of alkylation, which indicates that there is a certain weight fraction of alkyl chains, which is necessary for enhanced solubility. 
FTIR spectroscopy could also clearly prove the success of the reaction. Strong $\mathrm{C}-\mathrm{H}$ bands arise at $2900 \mathrm{~cm}^{-1}$ with increasing intensity, which can be clearly attributed to the alkyl chains. Finally, size exclusion chromatography (SEC) using NMP as eluent could also give a qualitative proof of the success of the grafting reaction. An increase of the apparent weight-average molecular weight was observed. As the shape and solvation state of the macromolecules is however changing with increasing functionalization, the SEC results cannot be discussed in detail and the SEC data should not be overstressed.

The thermal properties of the polymers were evaluated using thermogravimetric analysis (TGA). All polymers were stable up to $320^{\circ} \mathrm{C}$ under air atmosphere. Above this temperature, thermal degradation of the alkyl chains settled in. Full decomposition started around $500^{\circ} \mathrm{C}$. The weight fraction of alkyl as determined by TGA is compiled in Table 1 and generally in good agreement with the NMR results. Initial experiments on the potential microphase separation were performed on polymer powders using small-angle and wide-angle X-ray scattering (SAXS and WAXS, see Figure 2a, 2b). A microphase separation could be observed after thermal annealing at $240^{\circ} \mathrm{C}$ under $\mathrm{N}_{2}$ atmosphere. Only a weak peak at scattering vector $q=1.8-1.9 \mathrm{~nm}^{-1}$ was observed in the SAXS patterns for PBI-C $\mathrm{C}_{18}-1$ and -2 , while PBI$\mathrm{C}_{18^{-}} 3$ showed a more pronounced but still broad peak $\left(q=1.9 \mathrm{~nm}^{-1}\right)$, corresponding to a $d$-spacing of $\sim 3.3 \mathrm{~nm}$. No clear structure assignment could be made on the basis of the SAXS patters solely, as no other features could be identified clearly.

WAXS showed amorphous halos only, except for PBI- $\mathrm{C}_{18}-3$, for which weak reflections were observed at $2 \theta \sim 21.7$ and $24^{\circ}$, indicative of partially crystallized alkyl chains. Further indication of partial crystallinity was derived from scanning electron microscopy (SEM). No distinct morphology was observed for low D.A. (see Figure 2c). This is in contrast to PBI- $\mathrm{C}_{18}-3$, which showed some plate-like layered morphologies and even clear facets after annealing (Figure 2d). This leads to the conclusion, that upon high D.A., the polymers do show a significant microphase separation, most probably into a lamellar phase. The microphase separation comes along with crystallization of the long alkyl side-chains. This picture is in good agreement with reports on lamellar phases formed from polymers bearing long alkyl side chains, such as polyelectrolyte complexes or poly(ionic liquids) [21, 22].

Finally, we were interested whether the microphase separation is also effective upon doping with phosphoric acid. Membranes were cast from THF and annealed before proceeding further on. The membrane was cut into pieces and soaked in $\mathrm{H}_{3} \mathrm{PO}_{4}$ of various concentrations $(2 \mathrm{M}, 6 \mathrm{M}, 10 \mathrm{M}$ or $14 \mathrm{M}$, respectively). The membranes were dried at $80^{\circ} \mathrm{C}$ under vacuum afterwards and the acid uptake was determined gravimetrically (see Figure $4 b$ ). As expected, the uptake increased with increasing phosphoric acid concentration except for doping of PBI- $\mathrm{C}_{18}-3$ with $2 \mathrm{M} \mathrm{H}_{3} \mathrm{PO}_{4}$. This sample does show only very low acid uptake, this may be due to the hydrophobicity of the material, which could ham-

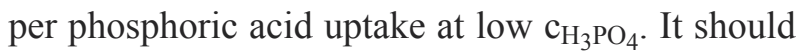
be noted that the membranes kept their shape very well during acid doping and drying.

The microphase separation was again analyzed by SAXS investigations (Figure 3a-3c). The observed peaks got much stronger (and sharper) with increasing acid uptake for all graft copolymers, indicating that the microphase separation got much stronger with increasing acid loading. Given the non-polar character of the $\mathrm{C}_{18}$ alkyl chains, this comes as no surprise. It can be suspected that the phosphoric acid populates the polar PBI domain (forming benzimidazolium salts and/or being hydrogen bonded). This results first of all in a swelling of the PBI domain that gives rise to larger d-spacings with increasing acid loading $(d \sim 4.4 \mathrm{~nm}$ after doping with $14 \mathrm{M} \mathrm{H}_{3} \mathrm{PO}_{4}$ compared to $d \sim 3.3 \mathrm{~nm}$ in the case of PBI- $\mathrm{C}_{18^{-}} 3$, see Figure 3d). Secondly, the segregation tendency is increased as a consequence of the increased hydrophobic-hydrophilic contrast. In analogy to block copolymer microphase-separation physics, it can be argued that the Flory-Huggins interaction parameter $\chi$ is changed (increased), which leads to stronger segregation tendency. Compared to the universal block copolymer phase-diagram, this could be interpreted as shift from the disordered phase into the lamellar regime.

Consequently, higher order reflections got visible in the case of PBI- $\mathrm{C}_{18}-2$ and -3 starting from acid loadings of $6 \mathrm{M}$. The higher order reflections were observed at $q \sim 2.8 \mathrm{~nm}^{-1}$, which is the double of the $q$-value of the primary peak ( $q \sim 1.4$ to $1.5 \mathrm{~nm}^{-1}$ ). Such $1: 2$ ratio is typically observed for lamellar structures. For PBI- $\mathrm{C}_{18}-1$, no higher order reflections got 

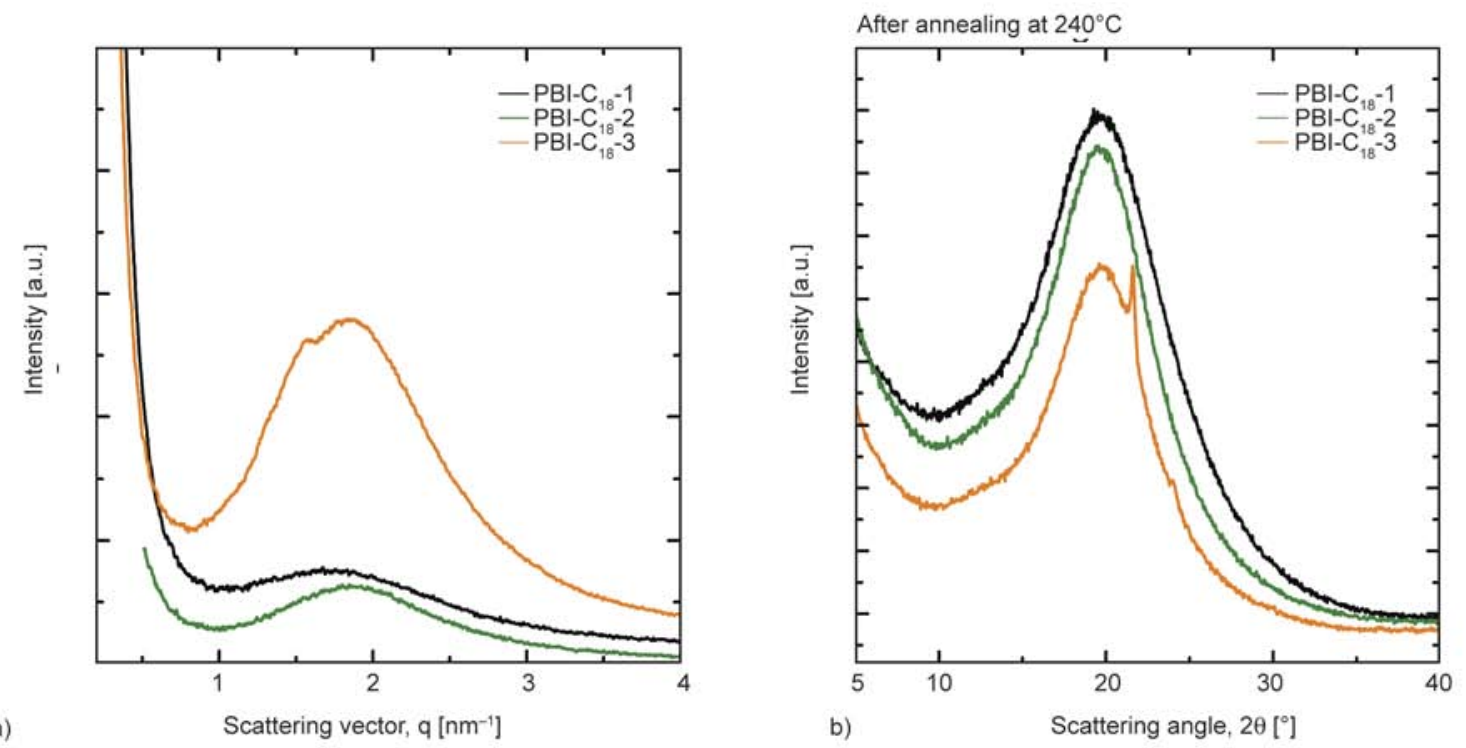

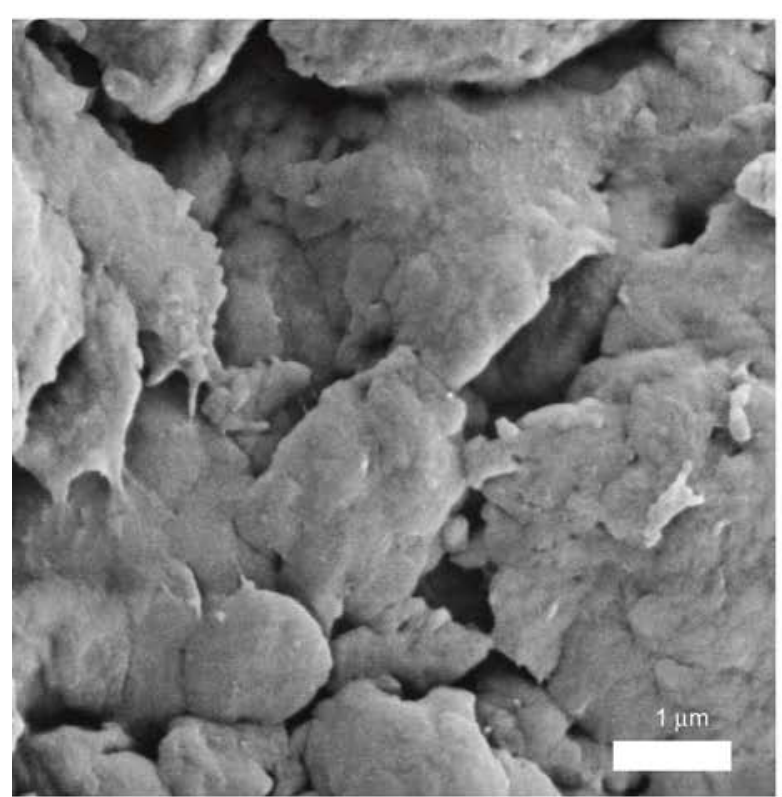

c)

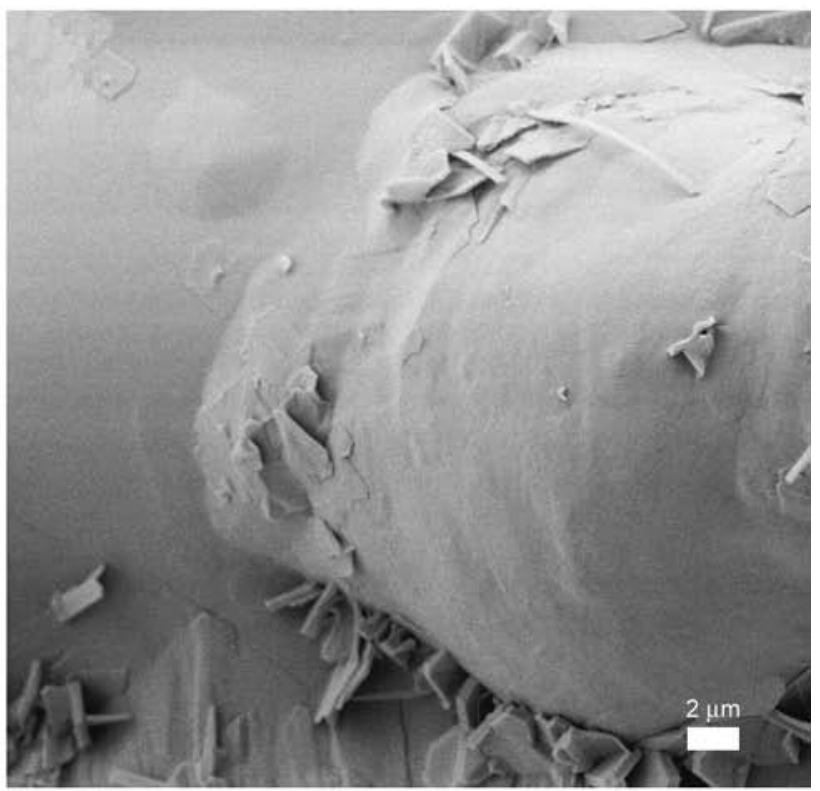

d)

Figure 2. SAXS (a) and WAXS (b) patterns of the modified PBIs (after annealing of the powders at $240^{\circ} \mathrm{C}$ ); SEM picture of annealed powders: $\mathrm{PBI}-\mathrm{C}_{18^{-}}-2$ (c) and $\mathrm{PBI}-\mathrm{C}_{18^{-}}-3$ (d)

visible clearly, probably as a consequence of the lower alkyl content and the reduced ability to undergo microphase separation into well-defined morphologies.

The observed peaks were rather broad in all cases. This can be interpreted as a consequence of smallsized ordered domains coexisting with a microphase-separated morphology of only short range order.

\subsection{Conductivity}

We analyzed the proton conductivity $\sigma$ of the phosphoric acid doped PBI graft copolymers prelimi- nary in order to get a first idea on the effect of the nanostructuration on $\sigma$. Hence, no temperature dependency was studied within this communication. For better comparability, we measured also the proton conductivity of a plain PBI membrane after phosphoric acid doping. This gives us some 'internal' standard as it is known that the conductivity of PA doped PBI can depend on the processing history (sol-gel doping, used solvent for membrane casting, gel methodology) [23-25]. The phosphoric acid doped membranes were dried under vacuum at $60^{\circ} \mathrm{C}$ for 16 hours before performing the measurements. The membranes were contacted between 

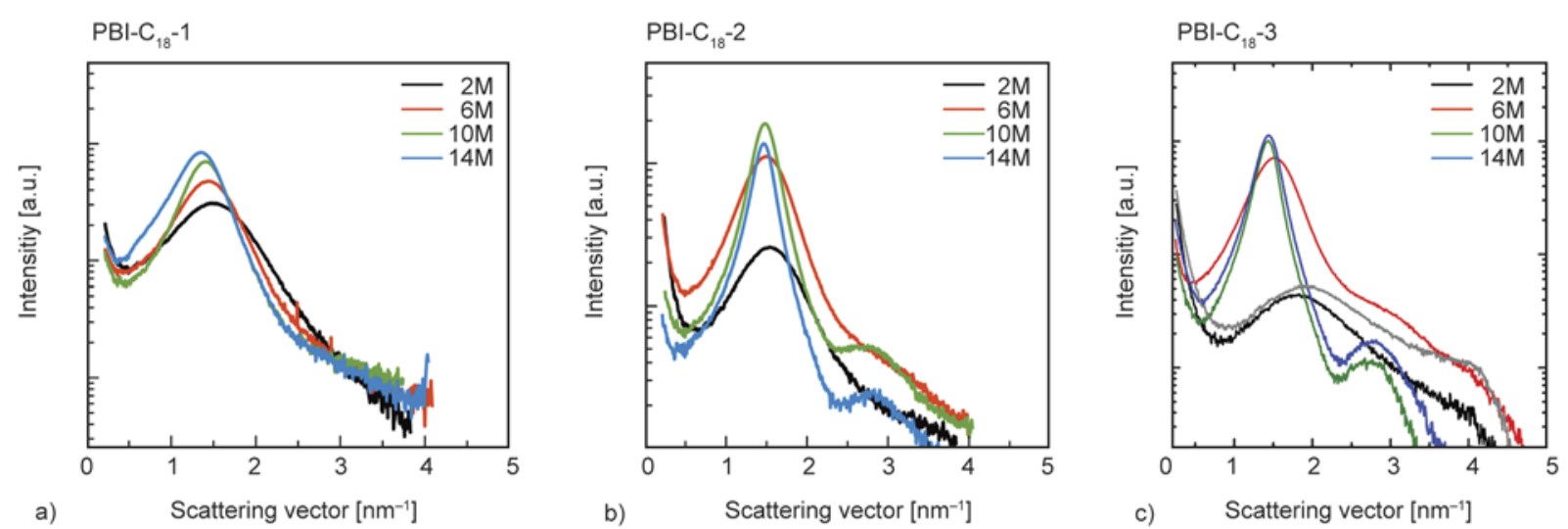

d)
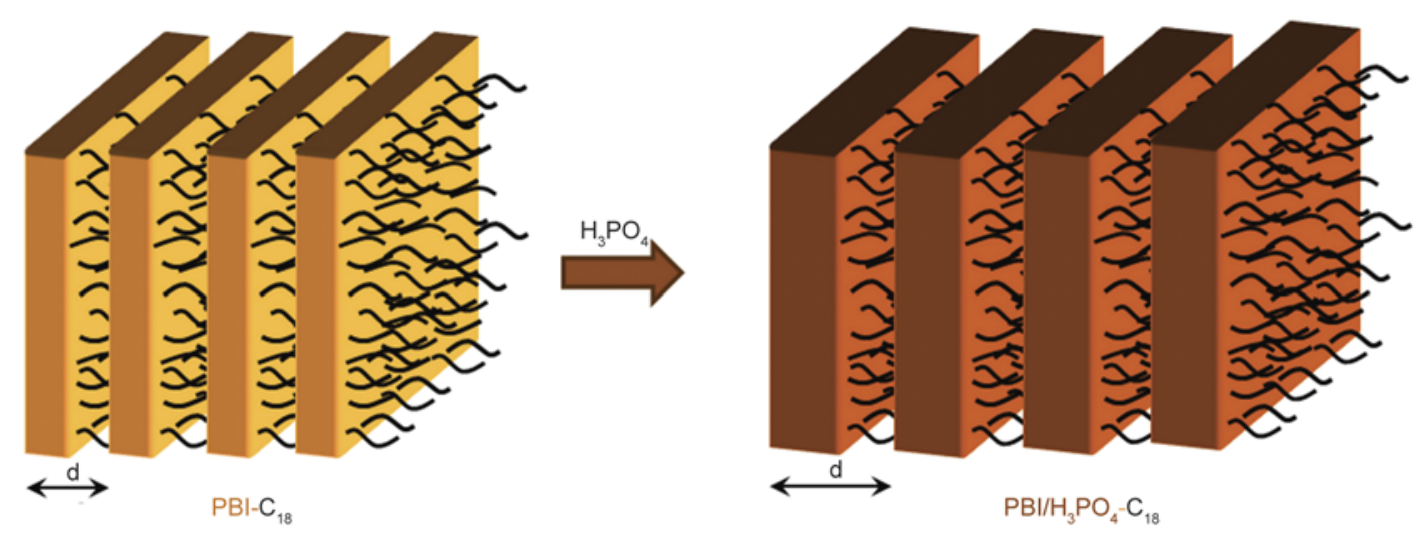

Figure 3. SAXS patterns of phosphoric acid doped PBI membranes: (a) PBI-C $18^{-1}$; (b) PBI-C ${ }_{18}-2$; (c) PBI-C ${ }_{18}-3$ and (d) schematic idealized drawing of the layer expansion upon acid doping

platinum foils and sealed to the external atmosphere; hence the measurements were done under rather low humidity $(<5-10 \%)$. The measurements were done using an impedance analyzer.

Figure $4 \mathrm{a}$ shows the proton conductivity of $\mathrm{PBI}$ and PBI- $\mathrm{C}_{18-3}-3$ in dependence of the acid doping. It

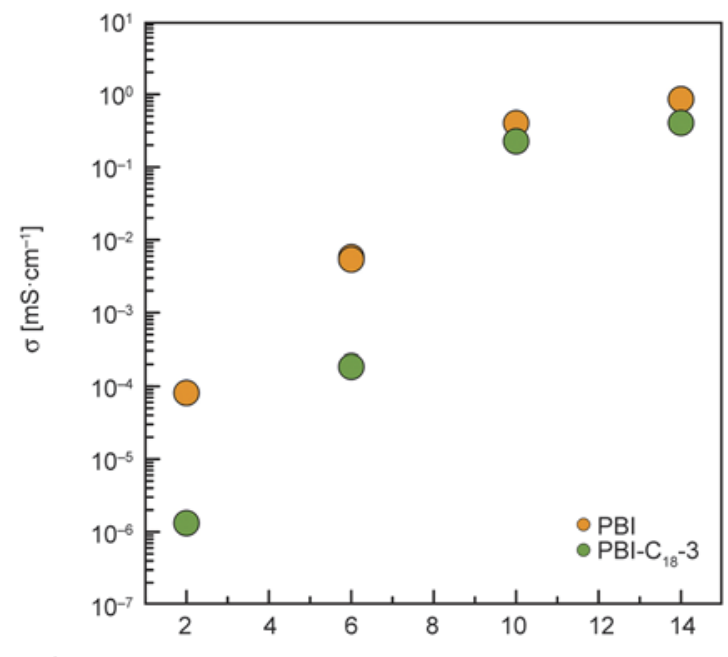

a)

Acid doping $[\mathrm{M}]$ becomes clear that the conductivity upon acid doping gets reduced if the PA is confined into a microphase-separated morphology. This is not surprising, as the total number of charge carriers get reduced upon grafting in accordance with the lower phosphoric acid uptake compared to plain PBI mem-

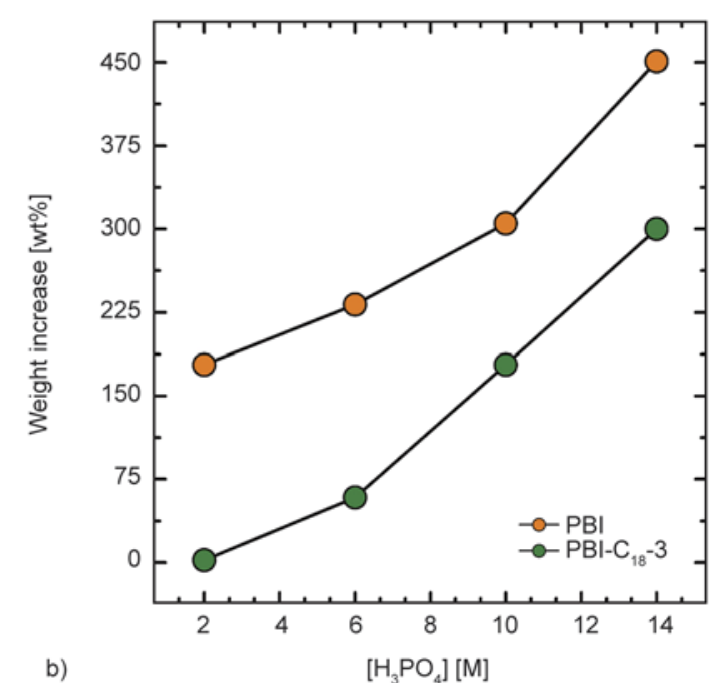

Figure 4. (a) proton conductivity of a pure PBI membrane and PBI-C $18-3$ in dependence of the acid doping level (measured after vacuum drying in a closed cell at room temperature $\left(22.4^{\circ} \mathrm{C}\right)$ relative humidity of the surrounding: $\left.16 \%\right)$; (b) weight increase of a PBI and PBI- $\mathrm{C}_{18}-3$ membrane upon doping with phosphoric acid and vacuum drying 
branes [26]. Nevertheless, the conductivity is in an acceptable range, which indicates that the PBI domains should be well connected. Furthermore, there are reports on increased proton hopping rates for doped ionic liquids, which are confined in microphase separated domains of block copolymers. The effect was believed to be related to a changed polymer morphology (chain stretching), which has some kind of back-coupling effects on the hydrogen-bond structure [26]. The results presented here can at the present stage however not give an indication, whether comparable effects could be also true in the modified and acid-doped PBI membranes. However, the preliminary results show in accordance with earlier reports that comparable proton conductivities can be reached in nanostructured PBI at lower PA content [10].

\section{Conclusions}

The present work shows that nanostructured PBI membranes can be obtained after $\mathrm{N}$-alkylation with long $\left(\mathrm{C}_{18}\right)$ alkyl chains. Polymers of different degrees of alkylation were prepared and characterized by a variety of methods. While the microphase separation is only weak at low degree of alkylation, it can be enhanced by larger amounts of incorporated alkyl chains. Membranes can be formed and doped with phosphoric acid. As a consequence of the non-polar alkyl chains the total acid uptake is lower compared to plain PBI. The acid populates the PBI domains, thereby increasing the segregation tendency between the non-polar alkyl chains and the PBI backbone. This results in the formation of lamellar domains as evidenced by SAXS experiments. The proton conductivity of the modified membranes was found to be lower than that of the pure PBI, but no detailed analysis has been carried out within this communication yet. Nevertheless, the present study opens the possibility to study the impact of the 2D like confinement of the phosphoric acid and the presence of a large amount of interfaces on the proton conductivity in more detail.

\section{References}

[1] Kreuer K. D.: On the development of proton conducting polymer membranes for hydrogen and methanol fuel cells. Journal of Membrane Science, 185, 29-39 (2001). DOI: $\underline{10.1016 / \mathrm{S} 0376-7388(00) 00632-3}$
[2] Kreuer K-D., Paddison S. J., Spohr E., Schuster M.: Transport in proton conductors for fuel-cell applications: Simulations, elementary reactions, and phenomenology. Chemical Reviews, 104, 4637-4678 (2004). DOI: $10.1021 / \mathrm{cr} 020715 \mathrm{f}$

[3] Gebel G., Diat O.: Neutron and X-ray scattering: Suitable tools for studying ionomer membranes. Fuel Cells, 5, 261-275 (2005).

DOI: $10.1002 /$ fuce. 200400080

[4] Schmidt-Rohr K., Chen Q.: Parallel cylindrical water nanochannels in nafion fuel-cell membranes. Nature Materials, 7, 75-83 (2008).

DOI: $10.1038 /$ nmat2074

[5] Wainright J. S., Wang J-T., Weng D., Savinell R. F., Litt M.: Acid-doped polybenzimidazoles: A new polymer electrolyte. Journal of the Electrochemical Society, 142, 121-123 (1995).

DOI: $10.1149 / 1.2044337$

[6] Asensio J. A., Sánchez E. M., Gómez-Romero P.: Proton-conducting membranes based on benzimidazole polymers for high-temperature PEM fuel cells. A chemical quest. Chemical Society Reviews, 39, 3210-3239 (2010).

DOI: 10.1039/b922650h

[7] Vilciauskas L., Paddison S. J., Kreuer K-D.: Ab initio modeling of proton transfer in phosphoric acid clusters. The Journal of Physical Chemistry A, 113, 91939201 (2009).

DOI: $10.1021 / j p 903005 r$

[8] Weber J., Kreuer K-D., Maier J., Thomas A.: Proton conductivity enhancement by nanostructural control of poly(benzimidazole)-phosphoric acid adducts. Advanced Materials, 20, 2595-2598 (2008).

DOI: $10.1002 / \mathrm{adma} .200703159$

[9] Weber J., Antonietti M., Thomas A.: Mesoporous poly (benzimidazole) networks via solvent mediated templating of hard spheres. Macromolecules, 40, 12991304 (2007). DOI: $10.1021 / \mathrm{ma} 062598 \mathrm{~m}$

[10] Lee H-S., Roy A., Lane O., McGrath J. E.: Synthesis and characterization of poly(arylene ether sulfone)- $b$ polybenzimidazole copolymers for high temperature low humidity proton exchange membrane fuel cells. Polymer, 49, 5387-5396 (2008). DOI: $10.1016 /$ j.polymer.2008.09.019

[11] Mader J. A., Benicewicz B. C.: Synthesis and properties of segmented block copolymers of functionalised polybenzimidazoles for high-temperature PEM fuel cells. Fuel Cells, 11, 222-237 (2011). DOI: $10.1002 /$ fuce. 201000085

[12] Ng F., Bae B., Miyatake K., Watanabe M.: Polybenzimidazole block sulfonated poly(arylene ether sulfone) ionomers. Chemical Communications, 47, 8895-8897 (2011).

DOI: $10.1039 / \mathrm{c} 1 \mathrm{cc} 12266 \mathrm{e}$ 
[13] Sivanandan K., Chatterjee T., Treat N., Kramer E. J., Hawker C. J.: High surface area poly(3-hexylthiophenes) thin films from cleavable graft copolymers. Macromolecules, 43, 233-241 (2010).

DOI: $10.1021 / \mathrm{ma902009q}$

[14] Gieselman M. B., Reynolds J. R.: Water-soluble polybenzimidazole-based polyelectrolytes. Macromolecules, 25, 4832-4834 (1992).

DOI: $10.1021 / \mathrm{ma} 00044 \mathrm{a} 056$

[15] Klaehn J. R., Luther T. A., Orme C. J., Jones M. G., Wertsching A. K., Peterson E. S.: Soluble N-substituted organosilane polybenzimidazoles. Macromolecules, 40, 7487-7492 (2007).

DOI: $10.1021 / \mathrm{ma} 062186 \mathrm{~d}$

[16] Kumbharkar S. C., Kharul U. K.: New $N$-substituted ABPBI: Synthesis and evaluation of gas permeation properties. Journal of Membrane Science, 360, 418425 (2010).

DOI: 10.1016/j.memsci.2010.05.041

[17] Maity S., Sannigrahi A., Ghosh S., Jana T.: N-alkyl polybenzimidazole: Effect of alkyl chain length. European Polymer Journal, 49, 2280-2292 (2013).

DOI: $10.1016 / j$.eurpolymj.2013.05.011

[18] Vogel H., Marvel C. S.: Polybenzimidazoles, new thermally stable polymers. Journal of Polymer Science, 50, 511-539 (1961). DOI: $10.1002 /$ pol.1961.1205015419

[19] Musto P., Karasz F. E., MacKnight W. J.: Fourier transform infra-red spectroscopy on the thermo-oxidative degradation of polybenzimidazole and of a polybenzimidazole/polyetherimide blend. Polymer, 34, 2934-2945 (1993).

DOI: $10.1016 / 0032-3861(93) 90618-\mathrm{K}$
[20] Conti F., Willibold S., Mammi S., Korte C., Lehnert W., Stolten D.: Carbon NMR investigation of the polybenzimidazole-dimethylacetamide interactions in membranes for fuel cells. New Journal of Chemistry, 37, 152-156 (2012).

DOI: $10.1039 / \mathrm{C} 2 \mathrm{NJ} 40728 \mathrm{~K}$

[21] Yuan J., Soll S., Drechsler M., Müller A. H. E., Antonietti M.: Self-assembly of poly(ionic liquid)s: Polymerization, mesostructure formation, and directional alignment in one step. Journal of the American Chemical Society, 133, 17556-17559 (2011). DOI: $10.1021 /$ ja207080j

[22] Thünemann A. F.: Polyelectrolyte-surfactant complexes (synthesis, structure and materials aspects). Progress in Polymer Science, 27, 1473-1572 (2002). DOI: $10.1016 / \mathrm{S} 0079-6700(02) 00017-5$

[23] Ma Y-L., Wainright J. S., Litt M. H., Savinell R. F.: Conductivity of PBI membranes for high-temperature polymer electrolyte fuel cells. Journal of the Electrochemical Society, 151, 8-16 (2004).

DOI: $10.1149 / 1.1630037$

[24] Xiao L., Zhang H., Scanlon E., Ramanathan L. S., Choe E-W., Rogers D., Apple T., Benicewicz B. C.: High-temperature polybenzimidazole fuel cell membranes via a sol-gel process. Chemistry of Materials, 17, 5328-5333 (2005).

DOI: $10.1021 / \mathrm{cm} 050831+$

[25] Sannigrahi A., Ghosh S., Maity S., Jana T.: Polybenzimidazole gel membrane for the use in fuel cell. Polymer, 52, 4319-4330 (2011).

DOI: $10.1016 /$ j.polymer.2011.07.013

[26] Hoarfrost M. L., Tyagi M. S., Segalman R. A., Reimer J. A.: Effect of confinement on proton transport mechanisms in block copolymer/ionic liquid membranes. Macromolecules, 45, 3112-3120 (2012).

DOI: $10.1021 / \mathrm{ma} 202741 \mathrm{~g}$ 